Phys. Rev. D 100, 023536 (2019)

arXiv:1903.10450

\title{
Regularized big bang singularity
}

\author{
F.R. Klinkhamer* \\ Institute for Theoretical Physics, \\ Karlsruhe Institute of Technology (KIT), \\ 76128 Karlsruhe, Germany
}

\begin{abstract}
Following up on earlier work on the regularization of the singular Schwarzschild solution, we now apply the same procedure to the singular Friedmann solution. Specifically, we are able to remove the divergences of the big bang singularity, at the price of introducing a 3-dimensional spacetime defect with a vanishing determinant of the metric. This particular regularization also suggests the existence of a "pre-big-bang" phase.

PACS numbers: 04.20.Cv, 98.80.Bp
\end{abstract}

Keywords: general relativity, big bang theory

*frans.klinkhamer@kit.edu 


\section{INTRODUCTION}

The Friedmann solution [1, 2] of an expanding universe has the so-called big bang singularity with diverging curvature, energy density, and temperature [the cosmic scale factor $a(t)$ drops to zero at cosmic time coordinate $\left.t=t_{\mathrm{bb}}=0\right]$. Quantum mechanical effects may temper these divergences; see, e.g., Refs. [3, 4] for two general discussions from different perspectives (loop quantum gravity and string theory, respectively).

Awaiting the definitive theory of "quantum gravity," we propose to remain within the domain of 4-dimensional general relativity but to allow for degenerate metrics. In fact, a particular degenerate metric has already provided a "regularization" of the Schwarzschild singularity [5] 10], removing the divergent behavior at the center (radial coordinate $r=0$ ) at the price of introducing a spatial 2-surface $(r=b>0)$ with a vanishing determinant of the metric. In the present article, we propose doing something similar with the big bang singularity (at cosmic time coordinate $\tau=0$, in a notation that will be explained later), by the introduction of a spatial 3-surface (at $|\tau|=b / c>0$ ) with a vanishing determinant of the metric. We emphasize, right from the start, that it is the differential structure (rather than the topology) which plays a crucial role for the regularization of the big bang singularity. Incidentally, the length scale $b$ may or may not be related to the Planck length [11].

With a suitable cosmic time coordinate $T=T(\tau) \in \mathbb{R}$ and an appropriate Ansatz for a degenerate metric, we obtain an odd solution for the cosmic scale factor $a(T)$, which "jumps" over the value $a=0$ and, thereby, avoids the big bang singularity. This particular T-odd solution may be of interest to the recent proposal for a $C P T$-symmetric universe [12]. There is also a $T$-even solution $a(T)$, which is strictly positive definite and, thus, stays away from the value $a=0$.

At this moment, it may be helpful to clarify what we mean by "general relativity." Standard general relativity is simply the theory as exposed by Einstein in his seminal 1916 article [13] and elaborated upon by various textbooks such as Refs. [2, 14]. In practical terms, the crucial element of general relativity is the Einstein gravitational field equation for the metric tensor $g_{\mu \nu}(x)$. But Einstein makes the further assumption (in Part B, Sec. 8 of Ref. [13]) that the determinant of the metric vanishes nowhere, $g(x) \equiv \operatorname{det} g_{\mu \nu}(x) \neq 0$, and the metric is said to be nondegenerate (see also Sec. 2.6 of Ref. [14]). All of this defines 
standard general relativity.

Einstein's nondegeneracy assumption is certainly reasonable under "normal" circumstances (with a metric "not too far away" from the Minkowski metric) but perhaps not under "unusual" circumstances, such as when spacetime singularities appear. We propose to consider, under these unusual circumstances, metrics which obey the standard Einstein equation but have a vanishing determinant over a submanifold of the spacetime manifold. (This submanifold may be considered to correspond to a "spacetime defect" [6 [10], as will be explained in Sec. III) In this sense, we use an extended version of general relativity by keeping the Einstein gravitational field equation but allowing for degenerate metrics. General relativity with degenerate metrics has been considered before; see, e.g., Ref. [15]. Degenerate effective metrics also appear in the context of condensed matter physics and are perhaps accessible by experiment [16, 17].

\section{STANDARD FLRW UNIVERSE}

Let us, first, review the main points of the standard spatially flat radiation-dominated Friedmann-Lemaître-Robertson-Walker (FLRW) universe. Details and further references

can be found in, e.g., Ref. [2]. Greek indices run over $\{0,1,2,3\}$ and Latin indices over $\{1,2,3\}$. Unless stated otherwise, we set $c=1$ and $\hbar=1$.

The line element of the standard spatially flat FLRW universe in comoving coordinates reads

$$
\begin{aligned}
\left.d s^{2}\right|_{\text {stand. FLRW }} & \left.\equiv g_{\mu \nu}(x) d x^{\mu} d x^{\nu}\right|_{\text {stand. FLRW }}=-d t^{2}+a^{2}(t) \delta_{k l} d x^{k} d x^{l}, \\
t & \in(0, \infty), \\
x^{k} & \in(-\infty, \infty),
\end{aligned}
$$

where the restricted range of the cosmic time coordinate $t$ will be explained shortly. The real function $a(t)$ corresponds to the cosmic scale factor. As far as the metric is concerned, the sign of $a(t)$ is irrelevant.

With this metric and the energy-momentum tensor of a homogeneous perfect fluid [energy density $\rho(t)$ and pressure $P(t)]$, the Einstein equation without a cosmological constant $\Lambda$ 
gives the spatially flat Friedmann equation [1] and the energy-conservation equation:

$$
\begin{aligned}
\left(\frac{1}{a(t)} \frac{d a(t)}{d t}\right)^{2} & =\frac{8 \pi}{3} G_{N} \rho(t), \\
\frac{d}{d a}\left[a^{3} \rho(a)\right]+3 a^{2} P(a) & =0,
\end{aligned}
$$

to which is added the equation of state,

$$
P=P(\rho)
$$

Consider, for definiteness, relativistic matter,

$$
P=\frac{1}{3} \rho
$$

so that (2.2b) implies $\rho \propto 1 / a^{4}$. The resulting cosmic scale factor from (2.2a) is then

$$
\left.a(t)\right|_{\text {stand. FLRW }} ^{(\text {rel-mat. sol. })}=\sqrt{t / t_{0}}
$$

For the particular solution (2.4), the zero point of the cosmic time coordinate $t$ has been shifted, so that

$$
\left.\lim _{t \rightarrow 0^{+}} a(t)\right|_{\text {stand. FLRW }} ^{(\text {rel-mat. sol. })}=0
$$

and $t=0$ corresponds to the big bang singularity. In addition, the cosmic scale factor (2.4) has been normalized to 1 at a given time $t=t_{0}>0$ for which the Hubble constant is assumed to be positive, $H_{0} \equiv[(d a / d t) / a]_{t=t_{0}}>0$.

The standard FLRW spacetime manifold with metric (2.1a) and cosmic scale factor (2.4) has the line element

$$
\left.d s^{2}\right|_{\text {stand. FLRW }} ^{(\text {rel-mat. sol. })}=-d t^{2}+\sqrt{t^{2} / t_{0}^{2}} \delta_{k l} d x^{k} d x^{l}
$$

where the metric component $\sqrt{t^{2} / t_{0}^{2}}$ can be simplified to $t / t_{0}$, because both $t$ and $t_{0}$ are positive. The metric of this spacetime manifold solves the Einstein equation for a homogeneous perfect fluid of relativistic matter, but the manifold is geodesically incomplete. Indeed, there is a big bang singularity at $t=0$ with diverging curvature (as shown by, for example, the Kretschmann curvature scalar $\left.K \equiv R^{\mu \nu \rho \sigma} R_{\mu \nu \rho \sigma}\right)$ and diverging matter energy density $\rho$ 
and temperature $\mathcal{T}$. Recall that, for the special case of relativistic matter, the Ricci curvature scalar $R \equiv g^{\mu \nu} R_{\mu \nu}$ vanishes identically. Specifically, these quantities are given by the following expressions:

$$
\begin{aligned}
& \left.R(t)\right|_{\text {stand. FLRW }} ^{(\text {rel-mat. sol. })}=6\left[\left(\frac{1}{a(t)} \frac{d a(t)}{d t}\right)^{2}+\frac{1}{a(t)} \frac{d^{2} a(t)}{d t^{2}}\right]=0, \\
& \left.K(t)\right|_{\text {stand. FLRW }} ^{\text {(rel-mat. sol.) }}=12\left[\left(\frac{1}{a(t)} \frac{d a(t)}{d t}\right)^{4}+\left(\frac{1}{a(t)} \frac{d^{2} a(t)}{d t^{2}}\right)^{2}\right] \propto 1 / t^{4}, \\
& \left.\rho(t)\right|_{\text {stand. FLRW }} ^{\text {(rel-mat. sol.) }} \propto 1 / a^{4}(t) \propto 1 / t^{2} \\
& \left.\mathcal{T}(t)\right|_{\text {stand. FLRW }} ^{(\text {rel-mat. sol. })} \propto 1 / \sqrt[4]{a^{4}(t)} \propto 1 / \sqrt[4]{t^{2}},
\end{aligned}
$$

where the final expression for the temperature can be simplified to $1 / \sqrt{t}$, because $t$ is positive. Observe that the temperature expression $\mathcal{T}(a) \propto\left[a^{4}\right]^{-1 / 4}$ in $(2.7 \mathrm{~d})$ follows directly from (2.7c) and the Stefan-Boltzmann law $\rho \propto \mathcal{T}^{4}$ (see also the discussion in the last paragraph of Box. 29.2 on p. 779 of Ref. [2]).

In view of the results (2.4) and (2.7), it is clear that this particular solution of the Einstein equation is only well behaved if the range of the cosmic time coordinate $t$ is restricted to the open half-line $\mathbb{R}^{+}$.

\section{MODIFIED FLRW UNIVERSE}

As mentioned in Sec. [1, it is possible to obtain a regularized version [5] of the singular Schwarzschild solution [2] by a simple procedure. The first step is to perform local surgery on Euclidean 3-space: the interior of a ball with radius $b$ is removed and antipodal points on the boundary of the ball are identified. The resulting 3 -space $\widetilde{M}_{3}$ is topologically nontrivial: $\widetilde{M}_{3} \simeq \mathbb{R} P^{3}-\{$ point $\}$, where $\mathbb{R} P^{3}$ is the 3 -dimensional real-projective plane. A proper solution of the Einstein equation requires suitable coordinates over $\widetilde{M}_{3}$ and an appropriate Ansatz for the metric.

The obtained regularized Schwarzschild solution has a spatial 2-surface (with topology $\mathbb{R} P^{2}$ ) over which the determinant of the metric vanishes. This spatial 2-surface embedded in spacetime may be interpreted as a $(2+1)$-dimensional "defect" of spacetime with topology 
$\mathbb{R} P^{2} \times \mathbb{R}$. At the location of the "spacetime defect," the standard elementary-flatness condition does not apply, and the equivalence principle is violated (cf. Appendix D in Ref. [5]). The appearance of this spacetime defect is, apparently, the price to pay for the absence of the Schwarzschild curvature singularity (having a degenerate metric evades certain singularity theorems; cf. Sec. 3.1.5 in Ref. [8]). The technical details of this regularization procedure can be found in Ref. [5], with further discussion of the differential structure in Ref. [7] and further discussion of the physics of this particular type of spacetime defect in Refs. [6, 9, 10]. Some details on the mathematics of general relativity with degenerate metrics appear in Chap. 3 of Ref. [8] (different mathematical aspects are discussed in Ref. [15]).

The idea, now, is to apply the same procedure to the singular FLRW solution of Sec. II, where the surgery will concern the cosmic time axis. First, the standard cosmic time coordinate $t>0$ is replaced by an extended coordinate $\tau \in \mathbb{R}$. Then, surgery on this 1 -space removes the open $\tau$ interval $(-b, b)$, for $b>0$, and identifies the antipodal points $\tau=-b$ and $\tau=b$. In this case, the resulting 1 -space $\widetilde{M}_{1}$ is topologically trivial: $\widetilde{M}_{1} \simeq \mathbb{R} P^{1}-\{$ point $\} \simeq$ $\mathbb{R}$. A sketch is given in Fig. 1, which may be considered to be the 1-dimensional analog of Fig. 1 in Ref. [5] for the 3-dimensional Schwarzschild construction. Note that we will use the same symbol $b$ for the parameter of the Schwarzschild solution and the one of the Friedmann solution, but these length scales can, in principle, be different. Remember that we have set $c=1$.

Next, define a suitable cosmic time coordinate $T$ (not to be confused with the temperature $\mathcal{T}$ of matter):

$$
\begin{aligned}
& T \equiv \begin{cases}+\sqrt[4]{\tau^{4}-b^{4}}, & \text { for } \tau \geq b, \\
-\sqrt[4]{\tau^{4}-b^{4}}, & \text { for } \tau \leq-b,\end{cases} \\
& \tau \in(-\infty,-b] \cup[b, \infty),
\end{aligned}
$$

where $b$ is assumed to be positive. The coordinate $T$ from (3.1a) covers the cosmic time axis (3.1b), with a unique value of $T$ for each point of the axis (cf. Fig. 11). Furthermore, the expression (3.1a) has the same mathematical structure as the Schwarzschild-construction expression (2.26) in Ref. [7], where $y$ must be replaced by $T$ and $\pm r$ by $\tau$ and where, following Endnote 18 of Ref. [7], the squares are replaced by quartic powers and the square roots by 


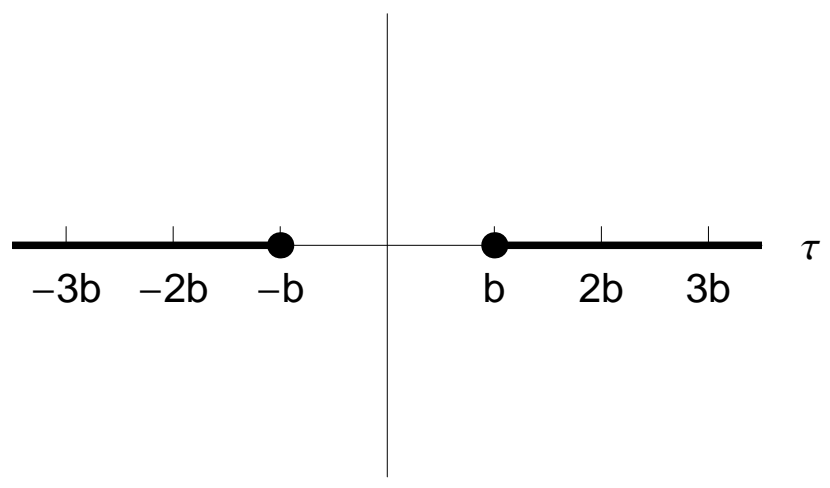

FIG. 1. Cosmic time axis $\tau \in(-\infty,-b] \cup[b, \infty)$, where the points $\tau=-b$ and $\tau=b$ are identified (as indicated by the dots).

fourth roots. It is, in principle, also possible to use $\widetilde{T} \equiv \pm \sqrt{\tau^{2}-b^{2}}$, but the choice (3.1a) has an advantage for the $T$-odd scale factor solution, as will be explained later.

With this new cosmic time coordinate $T \in \mathbb{R}$, we make the following Ansatz for the line element:

$$
\begin{aligned}
& \left.d s^{2}\right|_{\text {mod. FLRW }}=-\frac{T^{6}}{\left(b^{4}+T^{4}\right)^{3 / 2}} d T^{2}+a^{2}(\tau) \delta_{k l} d x^{k} d x^{l}, \\
& \left.a^{2}(\tau)\right|_{\tau=-b}=\left.a^{2}(\tau)\right|_{\tau=b}, \\
& T \in(-\infty, \infty), \\
& x^{k} \in(-\infty, \infty), \\
& \tau(T)= \begin{cases}+\sqrt[4]{b^{4}+T^{4}}, & \text { for } T \geq 0, \\
-\sqrt[4]{b^{4}+T^{4}}, & \text { for } T \leq 0,\end{cases}
\end{aligned}
$$

where the auxiliary coordinates $\tau=-b$ and $\tau=b$ correspond to the single point $T=0$ on the cosmic time axis (cf. Fig. 1). The particular form of the metric Ansatz (3.2a) is inspired by the metric of the modified Schwarzschild solution [5]. We notice that the coordinate transformation (3.2e is not a diffeomorphism, which is defined to be an invertible $\mathrm{C}^{\infty}$ function [14]. We also remark that, even for $a(\tau) \neq 0$, the metric from (3.2a) is degenerate: $\operatorname{det} g_{\mu \nu}=0$ at $T=0$. The corresponding $T=0$ spacetime slice may be interpreted as a 
3-dimensional "defect" of spacetime with topology $\mathbb{R}^{3}$. (For modified FLRW universes with positive or negative spatial curvature, the 3-dimensional defect of spacetime has topology $S^{3}$ or $\mathbb{H}^{3}$.)

It is straightforward to calculate the dynamic equations by evaluating the Einstein equation without a cosmological constant $\Lambda$ for the metric (3.2a with coordinates $\left\{T, x^{1}, x^{2}, x^{3}\right\}$ and the energy-momentum tensor of a homogeneous perfect fluid [energy density $\rho(T)$ and pressure $P(T)=\rho(T) / 3$ for the case of relativistic matter]. But the result also follows from the observation that the new metric (3.2a) written in terms of $\tau$ takes the same form as the standard metric (2.1a) in terms of $t$. The dynamic equations are, then, obtained if we replace $t$ with $\tau$ in (2.2) and change to the $T$ coordinate from (3.1a),

$$
\begin{aligned}
\left(1+\frac{b^{4}}{T^{4}}\right)^{3 / 2}\left(\frac{1}{a(T)} \frac{d a(T)}{d T}\right)^{2} & =\frac{8 \pi}{3} G_{N} \rho(T), \\
\frac{d}{d a}\left[a^{3} \rho(a)\right]+3 a^{2} P(a) & =0 \\
P(T) & =\frac{1}{3} \rho(T)
\end{aligned}
$$

where we have, again, considered relativistic matter. Compared to the standard FLRW equations (2.2a), (2.2b), and (2.3), the only difference in (3.3) is the Jacobian factor $(d T / d \tau)^{2}=$ $\left(1+b^{4} / T^{4}\right)^{3 / 2}$ on the left-hand side of the modified Friedmann equation (3.3a). This particular prefactor in (3.3a) allows for a solution $a(T)$ with $a(0) \neq 0$ and $[d a(T) / d T]^{2} \sim T^{6}$ near $T=0$. The modified Friedmann equation (3.3a) is, in fact, a singular differential equation (the singularity appears at $T=0$ ) with a nonsingular solution to be given shortly, whereas the standard Friedmann equation (2.2a) is a nonsingular differential equation with a singular solution (2.4).

The solutions $a(T)$ of (3.3) can be even or odd in $T$. In view of the recent interest [12] in a $T$-odd solution, we explicitly give our modified $T$-odd relativistic-matter solution $a(T)$ from (3.3),

$$
\left.a(T)\right|_{\text {mod. FLRW }} ^{(T \text {-odd rel-mat. sol. })}= \begin{cases}+\sqrt[8]{\left(b^{4}+T^{4}\right) /\left(b^{4}+T_{0}^{4}\right)}, & \text { for } T>0 \\ -\sqrt[8]{\left(b^{4}+T^{4}\right) /\left(b^{4}+T_{0}^{4}\right)}, & \text { for } T \leq 0\end{cases}
$$

with normalization $a\left(T_{0}\right)=1$ for $T_{0}>0$. Note that the solution (3.4) for $T>0$ reproduces, 
in the limit $b \rightarrow 0^{+}$and with the identification $T_{0}=t_{0}$, the positive- $t$ branch of the standard solution (2.4).

The solution (3.4) is discontinuous at $T=0$ but still has a monotonic behavior, $d a(T) / d T \geq 0$. Observe also that the $T$-odd solution (3.4) has continuous first-, second- , and third-order derivatives at $T=0$ and a discontinuous fourth-order derivative at $T=0$ (the derivatives must be defined appropriately). If the $T$ definition (3.1a) were replaced by $\widetilde{T} \equiv \pm \sqrt{\tau^{2}-b^{2}}$, the corresponding $\widetilde{T}$-odd solution $a(\widetilde{T})$ would already have a discontinuous second-order derivative at $\widetilde{T}=0$.

With a nonvanishing parameter $b$, the solution (3.4) gives finite values at $T=0$ for the Ricci curvature scalar $R$ (identically zero, in fact) and the Kretschmann curvature scalar $K$,

$$
\begin{aligned}
& R(T)\left.\right|_{\text {mod. FLRW }} ^{(\text {rel-mat. sol. })}=0 \\
&\left.K(T)\right|_{\text {mod. FLRW }} ^{(\text {rel-mat. sol. })}=\frac{3}{2} \frac{1}{b^{4}+T^{4}},
\end{aligned}
$$

and also for the matter energy density $\rho$ and the matter temperature $\mathcal{T}$,

$$
\begin{gathered}
\left.\rho(T)\right|_{\text {mod. FLRW }} ^{(\text {rel-mat. sol. })}=\rho_{0} \sqrt{\frac{b^{4}+T_{0}^{4}}{b^{4}+T^{4}}}, \\
\left.\mathcal{T}(T)\right|_{\text {mod. FLRW }} ^{\text {(rel-mat. sol. })}=\mathcal{T}_{0} \sqrt[8]{\frac{b^{4}+T_{0}^{4}}{b^{4}+T^{4}}},
\end{gathered}
$$

for finite boundary conditions $\rho_{0}>0$ and $\mathcal{T}_{0}>0$ at $T=T_{0}>0$ [the actual value of $\rho_{0}$, for given $T_{0}$, follows from (3.3a) and (3.4)]. The last result (3.5d) relies on the relation $\mathcal{T}(a) \propto\left[a^{4}\right]^{-1 / 4}$, as explained in the text below (2.7d $)$.

The maximum value of the Kretschmann scalar (3.5b) occurs at $T=0$ and is given by $K(0)=(3 / 2) b^{-4}$, which allows for the interpretation of the parameter $b$ from the metric Ansatz (3.2a) as the minimum curvature length scale of the resulting spacetime manifold. The maximum value of the matter density also occurs at $T=0$ and, from (3.3a) and (3.4), is given by $\rho(0)=(3 / 4) E_{\text {planck }}^{2} b^{-2}$, in terms of the reduced Planck energy $E_{\text {planck }} \equiv \sqrt{1 /\left(8 \pi G_{N}\right)} \approx 2.44 \times 10^{18} \mathrm{GeV}$. Similar results hold for a modified FLRW universe with nonrelativistic matter, as discussed in Appendix A. For completeness, we also present, in Appendix B, a particular modified FLRW universe with a positive cosmological constant $\Lambda$. 
For the record, we mention that the $T$-even relativistic-matter solution $a(T)$ has the same eighth roots as in (3.4) but now with a plus sign before both roots. This $T$-even solution is perfectly smooth at $T=0$. The results (3.5) hold also for the $T$-even solution.

We remark that it is possible to get the $T$-even solution in a somewhat simpler form if

we start from the definition $\widetilde{T}(\tau) \equiv \operatorname{sgn}(\tau) \sqrt{\tau^{2}-b^{2}}$ (cf. Endnote 18 in Ref. [7]), where the sign function is defined by $\operatorname{sgn}(x)=x / \sqrt{x^{2}}$ for $x \neq 0$ and $\operatorname{sgn}(x)=0$ for $x=0$. We then use the metric

$$
\left.d s^{2}\right|_{\text {mod. FLRW }} ^{(\widetilde{T} \text {-coord. })}=-\frac{\widetilde{T}^{2}}{b^{2}+\widetilde{T}^{2}} d \widetilde{T}^{2}+a^{2}(\tau) \delta_{k l} d x^{k} d x^{l}
$$

The corresponding modified Friedmann equation with relativistic matter now has the following $\widetilde{T}$-even solution:

$$
\left.a(\widetilde{T})\right|_{\text {mod. FLRW }} ^{(\widetilde{T} \text {-even rel-mat. sol. })}=\sqrt[4]{\left(b^{2}+\widetilde{T}^{2}\right) /\left(b^{2}+\widetilde{T}_{0}^{2}\right)} .
$$

For the rest of the discussion, we again focus on the $T$-odd solution (3.4).

\section{DISCUSSION}

In this article, we compare two spacetime manifolds. The first spacetime manifold corresponds to the standard FLRW universe with metric (2.1a) for an extended cosmic time coordinate $T \in \mathbb{R}$ and the following cosmic scale factor solution for the case of relativistic matter:

$$
\left.a(T)\right|_{\text {stand. FLRW }} ^{\text {(rel-mat. sol.) }}=\operatorname{sgn}(T) \sqrt[4]{T^{2} / T_{0}^{2}}
$$

which extends the previous solution (2.4) for positive cosmic time coordinate $t$. The line element of this first spacetime manifold is then given by

$$
\left.d s^{2}\right|_{\text {stand. FLRW }} ^{\text {(rel-mat. sol.) }}=-d T^{2}+\sqrt{T^{2} / T_{0}^{2}} \delta_{k l} d x^{k} d x^{l},
$$

with all spacetime coordinates $\left\{T, x^{1}, x^{2}, x^{3}\right\}$ ranging over $\mathbb{R}$. The Kretschmann curvature scalar $K(T)$, the matter energy density $\rho(T)$, and the matter temperature $\mathcal{T}(T)$ obtained from (4.1) are given by (2.7b), (2.7c), and (2.7d $)$ with $t$ replaced by $T$, and they are seen to diverge as $T \rightarrow 0$. The Einstein equation for the metric (4.2) is invalid at $T=0$ or, at least, ill defined. 
With the definition $\eta \equiv \pm 2\left[T^{2}\right]^{1 / 4}$, the line element (4.2) becomes conformally flat, $d s^{2}=(1 / 4) \eta^{2}\left[d \eta^{2}+\delta_{k l} d x^{k} d x^{l}\right]$, and corresponds to the background metric used in Ref. [12] with a slightly different notation. In terms of the conformal time $\eta$, the Kretschmann curvature scalar is given by $K(\eta) \propto 1 / \eta^{8}$, which diverges at $\eta=0$.

The second spacetime manifold corresponds to a modified FLRW universe with metric (3.2a and cosmic scale factor solution (3.4). The line element of this second spacetime manifold is given by

$$
\left.d s^{2}\right|_{\text {mod. FLRW }} ^{\text {(rel-mat. sol.) }}=-\frac{T^{6}}{\left(b^{4}+T^{4}\right)^{3 / 2}} d T^{2}+\sqrt[4]{\frac{b^{4}+T^{4}}{b^{4}+T_{0}^{4}}} \delta_{k l} d x^{k} d x^{l}
$$

with all spacetime coordinates ranging over $\mathbb{R}$. The metric (4.3) solves the Einstein equation with relativistic matter. The corresponding quantities $K(T), \rho(T)$, and $\mathcal{T}(T)$ are given by (3.5) and remain finite as $T \rightarrow 0$, provided the length parameter $b$ is nonvanishing.

Both of the above metrics are degenerate, det $g_{\mu \nu}=0$ at each spacetime point with $T=0$. Observe, however, that the metric from (4.3) at each spacetime point with $T=0$ has a single vanishing eigenvalue (provided $b \neq 0$ ), whereas the metric from (4.2) at the same spacetime point has three vanishing eigenvalues. Related to this last observation is the result that the Kretschmann curvature scalar $K$ is finite at $T=0$ for the metric (4.3) with nonzero $b$ but is singular at $T=0$ for the metric (4.2) [these results can be seen explicitly in (3.5b) for $b \neq 0$ and $b=0$, respectively]. Hence, the spacetime manifold (4.3), with a nonzero length parameter $b$ and a particular differential structure, may be considered to be a "regularized" version of the spacetime manifold (4.2). Corresponding results for a modified FLRW universe with a nonrelativistic matter component or a positive cosmological constant are given in Appendices $\mathrm{A}$ and $\mathrm{B}$.

We remark that, in general, a regularized theory may temporarily lose certain desirable properties, which are only recovered as the regulator is removed. An example is given by the lattice regularization of flat-spacetime quantum field theory, where the full Poincaréinvariance group is recovered in the continuum limit as the lattice spacing is taken to zero. Our regularized Friedmann solution is also far from perfect: the standard elementary-flatness condition breaks down at $T=0$, the location of the "spacetime defect" (cf. Appendix D in Ref. [5]). In addition, there is the $T=0$ discontinuity in the $T$-odd cosmic scale factor 
solution (3.4). This discontinuity disappears in the corresponding metric (4.3), so that scalar, vector, and tensor fields are unaffected by the $a(T)$ discontinuity at $T=0$. The spinor-field boundary conditions at $T=0^{+}$and $T=0^{-}$may require an appropriate $C P$ transformation. In any case, the metric (4.3) provides a spacetime manifold without curvature singularities, which allows for a meaningful study of the behavior of relativistic matter in the very early universe.

The previously considered spacetime defect of Refs. [5-10] resulted from surgery on space. Here, we have considered surgery on cosmic time. But it is also possible to skip the surgery discussion. The new metric Ansatz (3.2a) simply replaces the FLRW Ansatz (2.1a) and the resulting modified Friedmann equation (3.3a) gives a universe without a curvature singularity but with a 3-dimensional spacetime defect, which appears to be the "lesser evil."

It may be the case that the length parameter $b$ entering the classical metric (4.3) is not just a mathematical artifact ("regulator") but that it traces back to the underlying theory of "quantum spacetime." Even so, it is unclear whether or not the length scale $b$ is determined by the Planck length $l_{\text {planck }} \equiv \sqrt{8 \pi \hbar G_{N} / c^{3}} \approx 8.10 \times 10^{-35} \mathrm{~m}$, as the definitive quantumspacetime theory has not yet been established. See Ref. [11] for a general discussion of a possible fundamental length scale that is different from the Planck length.

Leaving aside a possible physical origin of the nonvanishing length parameter $b$ in the metric (4.3), we observe that the corresponding spacetime manifold is geodesically complete, as long as the cosmic time coordinate $T$ has an extended range, $T \in \mathbb{R}$. This manifold, then, has a pre-bounce phase for $T \leq 0$ (a "pre-big-bang" phase, in standard terminology), which may or may not have produced relics in the present post-bounce universe for $T>0$ (the present "post-big-bang" universe, in standard terminology).

In closing, we return to the condensed-matter-physics analogy mentioned in the last sentence of Sec. II. In superfluid ${ }^{3} \mathrm{He}$ experiments, there occur phase transitions between different topological phases [16, 17]. In one of these phases - the polar phase - the determinant of the effective tetrad field vanishes. This allows for a transition between two effective spacetimes with opposite chirality if the system starts in the polar distorted A-phase, moves into the polar phase, and then returns back (cf. Fig. 1 in Ref. [17]). The combined process in superfluid ${ }^{3} \mathrm{He}$ (two phase transitions and the intermediate polar phase) is analogous to the 
spacetime defect of the regularized big bang singularity, which has a vanishing determinant of the spacetime metric.

In superfluid ${ }^{3} \mathrm{He}$, the system passes between the effective spacetimes via two subsequent phase transitions and an intermediate phase, as the temperature is changed by hand. In cosmology, the universe passes from one phase (pre-bounce) to another phase (post-bounce) via the spacetime defect, as the universe evolves forward by the reduced Einstein equations with appropriate initial conditions in the pre-bounce phase.

\section{ACKNOWLEDGMENTS}

We thank G.E. Volovik for pointing out Ref. [12] and subsequent discussions, and J.M. Queiruga and Z.L. Wang for useful comments on the manuscript. Furthermore, we thank the referee for the suggestion to consider the case of a positive cosmological constant.

\section{Appendix A: Modified FLRW universe with nonrelativistic matter}

In this appendix, we give some results for the modified spatially flat FLRW universe with nonrelativistic matter instead of the relativistic matter considered in Sec. III Specifically, the equation of state $(3.3 \mathrm{c})$ is replaced by

$$
P(T)=0,
$$

where $T$ is the cosmic time coordinate from (3.1).

The modified $T$-odd nonrelativistic-matter solution $a(T)$ from (3.3a), (3.3b), and (A1) is given by

$$
\left.a(T)\right|_{\text {mod. FLRW }} ^{(T \text {-odd nonrel-mat. sol. })}= \begin{cases}+\sqrt[6]{\left(b^{4}+T^{4}\right) /\left(b^{4}+T_{0}^{4}\right)}, & \text { for } T>0 \\ -\sqrt[6]{\left(b^{4}+T^{4}\right) /\left(b^{4}+T_{0}^{4}\right)}, & \text { for } T \leq 0\end{cases}
$$

with normalization $a\left(T_{0}\right)=1$ for $T_{0}>0$. The corresponding expressions for the Ricci curvature scalar $R$ and the Kretschmann curvature scalar $K$ are

$$
\begin{aligned}
\left.R(T)\right|_{\text {mod. FLRW }} ^{\text {(nonrel-mat. sol.) }} & =\frac{4}{3} \sqrt{\frac{1}{b^{4}+T^{4}}}, \\
\left.K(T)\right|_{\text {mod. FLRW }} ^{\text {(nonrel-mat. sol.) }} & =\frac{80}{27} \frac{1}{b^{4}+T^{4}} .
\end{aligned}
$$


Both curvature scalars are nonsingular at $T=0$ for $b \neq 0$ and singular at $T=0$ for $b \rightarrow 0$.

Finally, the modified FLRW spacetime manifold with metric (3.2a) and cosmic scale factor solution (A2) has the following line element:

$$
\left.d s^{2}\right|_{\text {mod. FLRW }} ^{\text {(nonrel-mat. sol.) }}=-\frac{T^{6}}{\left(b^{4}+T^{4}\right)^{3 / 2}} d T^{2}+\left[\frac{b^{4}+T^{4}}{b^{4}+T_{0}^{4}}\right]^{1 / 3} \delta_{k l} d x^{k} d x^{l},
$$

with all spacetime coordinates ranging over $\mathbb{R}$.

For completeness, the $T$-even solution $a(T)$ has the same sixth roots as in (A2) but now with a plus sign before both roots. This $T$-even solution is perfectly smooth at $T=0$. The results (A3) and (A4) hold also for the $T$-even solution. Again, it is possible to get the $T$-even solution in a somewhat simpler form if we start from the definition $\widetilde{T} \equiv \operatorname{sgn}(\tau) \sqrt{\tau^{2}-b^{2}}$.

\section{Appendix B: Modified FLRW universe with a positive cosmological constant}

In this appendix, we give some results for the modified spatially flat FLRW universe with a positive cosmological constant $\Lambda$, which corresponds to a perfect fluid with constant vacuum energy density $\rho_{V}=\Lambda$ and pressure $P_{V}=-\Lambda$. The metric is again taken as (3.2a) for the cosmic time coordinate $T \in \mathbb{R}$.

The dynamic equations are now given by

$$
\begin{gathered}
\left(1+\frac{b^{4}}{T^{4}}\right)^{3 / 2}\left(\frac{1}{a(T)} \frac{d a(T)}{d T}\right)^{2}=\frac{8 \pi}{3} G_{N} \rho_{V}, \\
\frac{d}{d a}\left[a^{3} \rho_{V}\right]+3 a^{2} P_{V}=0 \\
P_{V}=-\rho_{V}=-\Lambda<0
\end{gathered}
$$

where $(\underline{\mathrm{B} 1 \mathrm{~b}})$ is satisfied automatically for the equation of state (B1c). With the following boundary conditions at $T_{0}>0$ :

$$
\begin{aligned}
a\left(T_{0}\right) & =1, \\
{[(d a / d T) / a]_{T=T_{0}} } & >0,
\end{aligned}
$$

the $T$-even solution of $(\mathrm{B} 1 \mathrm{a})$ reads

$$
\begin{aligned}
\left.a(T)\right|_{\text {mod. FLRW }} ^{(T \text {-even CC sol. })} & =\exp \left[H_{\mathrm{dS}}\left(\sqrt[4]{b^{4}+T^{4}}-\sqrt[4]{b^{4}+T_{0}^{4}}\right)\right], \\
H_{\mathrm{dS}} & \equiv \sqrt{8 \pi G_{N} \Lambda / 3}
\end{aligned}
$$


We remark that the standard FLRW solution from a positive cosmological constant, with $a(T) \propto \exp \left[H_{\mathrm{dS}} T\right]$ for $T \in(-\infty, \infty)$, has no big bang singularity at a finite value of $T$ and, hence, no need for regularization. Still, solution (B3al) may be of interest in that it joins an expanding de-Sitter-type phase to a contracting de-Sitter-type phase, with a spacetime defect in between. In fact, it is known that the complete de Sitter spacetime [14] contains a patch with an expanding spatially flat FLRW universe and another patch with a contracting spatially flat FLRW universe and that the quantum fields in one patch may have unexpected interactions with those in the other patch [18]. These two FLRW patches of the standard de Sitter spacetime have $a=0$ where they meet (the de Sitter manifold is, nevertheless, perfectly smooth everywhere), whereas the solution (B3) has an exponentially small but nonzero value of $a(T)$ at $T=0$, corresponding to the position of the spacetime defect.

[1] A.A. Friedmann, "Über die Krümmung des Raumes" (On the curvature of space), Z. Phys. 10, 377 (1922); “Über die Möglichkeit einer Welt mit konstanter negativer Krümmung des Raumes" (On the possibility of a world with constant negative curvature), Z. Phys. 21, 326 (1924).

[2] C.W. Misner, K.S. Thorne, and J.A. Wheeler, Gravitation (Princeton University Press, Princeton, NJ, 2017).

[3] A. Ashtekar, "The Big Bang and the quantum," AIP Conf. Proc. 1241, 109 (2010), arXiv:1005.5491.

[4] G. Veneziano, "A quantum universe before the Big Bang(s)?," J. Phys. Conf. Ser. 880, 012001 (2017).

[5] F.R. Klinkhamer, "A new type of nonsingular black-hole solution in general relativity," Mod. Phys. Lett. A 29, 1430018 (2014), arXiv:1309.7011.

[6] F.R. Klinkhamer, "Skyrmion spacetime defect," Phys. Rev. D 90, 024007 (2014), arXiv:1402.7048.

[7] F.R. Klinkhamer and F. Sorba, "Comparison of spacetime defects which are homeomorphic 
but not diffeomorphic," J. Math. Phys. (N.Y.) 55, 112503 (2014), arXiv:1404.2901,

[8] M. Guenther, "Skyrmion spacetime defect, degenerate metric, and negative gravitational mass," Master Thesis, KIT, September 2017; available from https://www.itp.kit.edu/en/ publications/diploma

[9] F.R. Klinkhamer and J.M. Queiruga, "Antigravity from a spacetime defect," Phys. Rev. D 97, 124047 (2018), arXiv:1803.09736.

[10] F.R. Klinkhamer, "On a soliton-type spacetime defect," arXiv:1811.01078 [J. Phys. Conf. Ser. (to be published)].

[11] F.R. Klinkhamer, "Fundamental length scale of quantum spacetime foam," JETP Lett. 86, 73 (2007), arXiv:gr-qc/0703009.

[12] L. Boyle, K. Finn, and N. Turok, "CPT-symmetric Universe," Phys. Rev. Lett. 121, 251301 (2018), arXiv:1803.08928.

[13] A. Einstein, "Die Grundlage der allgemeinen Relativitätstheorie" (The foundation of the general theory of relativity), Annalen Phys. (Leipzig) 49, 769 (1916).

[14] S.W. Hawking and G.F.R. Ellis, The Large Scale Structure of Space-Time (Cambridge University Press, Cambridge, England, 1973).

[15] G.T. Horowitz, "Topology change in classical and quantum gravity," Class. Quant. Grav. 8, 587 (1991).

[16] J. Nissinen and G.E. Volovik, "Dimensional crossover of effective orbital dynamics in polar distorted ${ }^{3}$ He-A: Transitions to antispacetime," Phys. Rev. D 97, 025018 (2018), arXiv:1710.07616.

[17] J.T. Mäkinen, V.V. Dmitriev, J. Nissinen, J. Rysti, G.E. Volovik, A.N. Yudin, K. Zhang, and V.B. Eltsov, "Half-quantum vortices and walls bounded by strings in the polar-distorted phases of topological superfluid ${ }^{3}$ He," Nature Commun. 10, 237 (2019), arXiv:1807.04328.

[18] D. Krotov and A.M. Polyakov, "Infrared sensitivity of unstable vacua," Nucl. Phys. B 849, 410 (2011), arXiv:1012.2107. 